\title{
The insulin - exercise connection
}

\section{Book review}

The late twentieth century Western world has achieved the most sedentary lifestyle for the mass of humanity in all human history. Our sedentary modern world also provides a glutton's feast of cheap sugar-and starch-rich breads, chips, pastas, cakes, cookies, candy, etc. so abundantly available that even those on welfare can afford to feast on these hyperinsulinemia-promoting carbo-riches. It is perhaps no coincidence that in order to rapidly (and cheaply) fatten cattle and hogs before slaughter, they are confined in crowded feed-lots where the animals have virtually no room to move, while being fed all the CHO-rich grain they can eat.

Modern obese humans routinely suffer from the unique twentieth century "disease" - hypokinesis - i.e. too little bodily movement. The late twentieth century Western epidemic of obesity is as much due to widespread chronic hypokinesis, as it is to the $\mathrm{CHO} /$ caloric excess typical of modern humans. Thus Thompson and colleagues note: "Body fat is significantly affected by a program of prescribed exercise in both sexes at all age levels.... Exercise has been shown to produce body fat loss without caloric restriction in both animals... and humans..., although the loss is usually more pronounced with caloric restriction.

In fact, reductions in activity level are strongly correlated with body fat increases, even if caloric intake is significantly reduced.... In addition, exercise decreases storage fat rather than LBM [lean body mass], whereas dietary interventions [i.e. dieting [tend to reduce both [body fat and LBM]."

Studies done in the 1970's with both men and women found that significant body fat loss could be produced simply through a regular (i.e. at least four days/week) long-term walking program, without any dieting. "Vigorous regular walking has resulted in reduced body fat stores, reduced... insulin requirements (a $36 \%$ decrease in the ratio of insulin/glucose concentration occurred), and [spontaneously] reduced food intake." A key feature of the essentiality of moderate aerobic exercise, i.e. walking (the primary "natural" form of "exercise" engaged in of necessity by virtually all of humanity prior to the twentieth century) to preventing/reducing obesity is that "exercise increases insulin sensitivity and decreases insulin resistance)".

The reason for this is quite simple. Actively exercising muscles may take in up to 30times more blood sugar than they do when at rest, and this cellular uptake of glucose occurs without insulin! Thus walking provides the body with an alternative method to remove excess glucose from the bloodstream without the usual need for insulin secretion. Taking a brisk long walk 30-60minutes after a large meal may help blunt the otherwise inevitable massive insulin surge large (CHO-rich) meals normally induce.

\section{The anti-insulin program}

i. Seriously reduce (better yet, eliminate) from the diet all processed, refined, junk food, high sugar (sucrose, fructose, glucose), high white flour "foods": bread, pasts, cake, pie, candy, ice cream, crackers, cereal, corn/potato chips, snack bars, waffles/pancakes, soft drinks, doughnuts, sweet syrups, ad infinitum.
Volume II Issue I - 2018

\author{
Tony Salvitti \\ Old Dominion University, USA
}

Correspondence: Tony Salvitti, Old Dominion University, USA, Email worldbodybuilding@live.com

Received: November 05, 2017 | Published: February 15, 2018

ii. Minimize intake of salt, especially salty CHO-foods: pretzels, chips, crackers, etc. "Salt increases plasma glucose and insulin response to starchy foods."

iii. Increase glucagon - stimulating with lean protein: low-fat (ideally range-fed, organic) beef, lamb, chicken, turkey, fish etc.

iv. Reduce CHO-intake from the typical American/British levels of 250-400grams/day to 75-150grams/day. These carbohydrates should be mainly vegetables, with small amounts of brown rice, millet, beans, almonds, pumpkin seeds and other unrefined, high-fibre natural foods.

v. Take 40-60minute brisk walks, 4-6days/week. Avoid walking in highly polluted areas and/or times of day, as toxins from auto exhaust may inhibit mitochondrial burning of fuel (i.e. fat) for energy.

vi. Take various supplements discussed in this article - Vitamin C, B6, B3, Zinc, Magnesium, and GLA.

\section{Additional nutritional/pharmacologic aids to fat loss/ insulin reduction}

\section{Chromium picolinate}

This form of chromium is well absorbed, and has been shown in various animal and human studies to aid in fat loss while at least modestly enhancing lean body mass. "The ability of chromium picolinate to enhance insulin responsiveness has been demonstrated in rat myoblast cell cultures. 72 -h pre-incubation with chromium picolinate (50ng $\mathrm{Cr} / \mathrm{ml}$ ) resulted in a $60 \%$ increase in insulin binding, and markedly enhanced glucose and leucine uptake". Dosage: $200 \mathrm{mcg}$ Chromium (as picolinate) two or three times daily for women; $200 \mathrm{mcg}$ three times daily or $400 \mathrm{mcg}$ twice daily for men.

Obesity, aging, chronic dieting, genetics, lack of exercise and lack of cold exposure may all lead to "subclinical" hypothyroidism, often involving deficient conversion of less active T4 to T3. T3 decreases the activity of D5D, reducing pro-insulin PGE2, just as do glucagon and EPA. T3 also stimulates fat burning. Ideally one should use T3 (Cytomel) only under a physician's care and guidance, but those who fit the low-thyroid profile and suffer from chronic obesity and fatigue, and who are willing to take practical, moral and legal responsibility for their own actions, may wish to experiment with modest doses of T3 - i.e., 2-3 mcg once or twice daily, taken morning and/or early afternoon. T3 is fast/short-acting, and most effects will be gone within 24 hours or less. Nonetheless, there is some risk here - caveat emptor! 
Heart palpitations, excessive sweating, racing thoughts, headaches, irritability, and insomnia are all hints - it's not for you! Those with known or suspected (past or present) hyperthyroidism, even if obese, should not use T3 without a doctor's care. Similarly those with any other serious disease states - especially heart arrhythmia's/heart disease - should be extremely cautious in T3 use.

\section{Anti-cortisol states}

Since cortisol levels tend to increase with age (and stress), and since cortisol promotes both obesity and insulin resistance, this is a key strategy to normalize weight/insulin levels. DHEA and high dose vitamin C may all help lower elevated cortisol levels. DHEA: 1050mg A.M. Gerovital-H3®: 100mg A.M. Dilantin (Phenytoin) 2550mg at bedtime. Vitamin C: 500-1000mg 3-4 times daily.

\section{L-Tryptophan/5-Hydroxytryptophan (Oxitriptan)}

Several human studies with 5HTP, the precursor of serotonin, have found good weight loss results with 5HTP. There is evidence that some humans compulsively snack on $\mathrm{CHO}$ foods to feel better. The large insulin releases generated by such "carbo-bingeing" preferentially increase tryptophan/serotonin in the brain, temporarily reducing anxiety and depression in such people. By providing an alternative, non-insulin-driven way to increase brain serotonin, L-Tryptophan, supplements may help reduce weight not only by reducing total caloric intake, but especially by reducing $\mathrm{CHO}$ intake, thus lessening hyper-insulinemia/insulin resistance. In the 1992 Italian study, $300 \mathrm{mg} / 5 \mathrm{HTP}$ supplements may help reduce weight not only by reducing total caloric intake, but especially by reducing $\mathrm{CHO}$ intake, thus lessening hyperinsulinemia/insulin resistance. In the 1992 Italian study, 300mg 5HTP 3 times daily before meals reduced women's caloric intake over a twelve week period from $3232 \mathrm{cal} /$ day to $1273 \mathrm{cal} /$ day, while reducing $\mathrm{CHO}$ intake from $350 \mathrm{gm} /$ day to $150 \mathrm{gm} /$ day. Weight dropped an average of eleven pounds. (The study did use special enteric-coated 5HTP capsules to prevent gut irritation). Taking 1000-1500mg L-Tryptophan at bedtime, or 50-100mg 5HTP before meals may reduce $\mathrm{CHO}$-craving and intake.

\section{Pro-GH supplements}

As noted earlier, PGE1 may enhance GH release. So all the PGE1enhancing nutrients (GLA, EPA, B3, B6, C, zinc, magnesium) may be helpful here. Hydergine ${ }^{\circledR}$ has been shown to increase GH-release in the elderly with long-term usage at $1.5 \mathrm{mg}$ every 6 hours. The authors of this study also note that bromocryptine (Parlodel) may also enhance adult GH-release. They also note that the enhanced pituitary GH-release from hydergine ${ }^{\circledR}$ seems to be related to an increase in brain (hypothalamic) dopamine status, which normally declines (often precipitously) with age. Thus the dopamine-enhancing agent Deprenyl may also be useful as part of a GH-restoration program.

\section{Mitochondrial energizers and protectants}

In a healthy human, storage fat is at a minimum and sooner or later all fat-dietary, body-manufactured, and storage fat - ends up as "fuel for the furnace" - i.e. the trillions of mitochondrial "power plants" found in most of our cells. Vitamins B1, B2, B3, B5 (pantothenic acid), and biotin, as well as NADH, alpha-lipoic acid, CoQ10/ Idebenone, magnesium and manganese are all necessary "spark plugs" to facilitate burning fat and sugar for energy. 10-100mg B1, B2, B3, 50-200mg B5, 1-10mg biotin, 5-20mg NADH, 50-300mg alpha-lipoic acid, 60-300mg CoQ10 and/or 45-135mg Idebenone, 200-500 mg magnesium, and 3-10mg managanese may optimize mitochondrial energy cycles. Since the mitochondrial structures inevitably generate massive amounts of free radicals in turning fuel into energy, and since these structures are rich in easily rancidified polyunsaturated fatty acids, a panoply of antioxidants - e.g. 100-400 IU vitamin E, 500-2000mg vitamin C, 100-200mcg selenium, 50$300 \mathrm{mg}$ alpha-lipoic acid, 500-1000mg N-acetylcysteine, $2 \mathrm{mg}$ copper as copper sebacate (SOD-mimetic), $50-100 \mathrm{mg}$ grape seed extract/ pycnogenol, 300-500mg silymarin - may help protect the essential "fat burning furnaces." In addition, 1gm L-carnitine twice daily on an empty stomach may facilitate fat burning - carnitine is the "shuttle molecule" that "escorts" fatty acids into mitochondria where they are then oxidized. ALC (acetyl-1-carnitine) may also be a useful mitochondrial regenerator - mitochondria become progressively deformed and dysfunctional with aging. Dosage: 1-3gms/day. Ward Dean suggests this dose can be half L-carnitine and half ALC to achieve successful mitochondrial regeneration.

\section{Caffeine}

Caffeine, whether from coffee or as a "drug", has many benefits for aiding fat loss. However, excessive doses (probably 300mg/ day and up, on average) may pose risks of "caffeinism", with such symptoms as headaches, restlessness, irritability, insomnia, anxiety, excessive urination, gut irritation, heart palpitations, and muscle tremors. A thermogenic/fat burning dose is probably $100-200 \mathrm{mg}$ daily - i.e. the equivalent of one to two cups of coffee/day, or two to four cups made with half decaf and half regular. Caffeine taken with a meal may induce increased thermogenesis-burning fat to make heat. It may increase resting metabolic rate-our resting metabolism burns $60-70 \%$ of our total daily energy consumption. Caffeine preadministration 45-60minutes before exercise has been shown to spare liver/muscle glycogen and to enhance fatty acid burning in humans. Caffeine taken after at least an eight hour fast, i.e. in the morning after arising, may be especially effective when combined with a 40-60minute brisk walk, to enhance burning of stored body fat.

\section{Biography}

Tony Salvitti is originally from Los Angeles, California, and attended and graduated high school in Kaiserslautern, in Vogelweh West Germany. Where he was trained in Muay Thai-competing against U.S. Army soldiers in the light heavyweight division (later becoming a heavyweight in natural bodybuilding at 230 ripped lbs.), hatha yoga, Judo, and lettered in track and field. From there he went to college, before being recruited in the U.S. Navy in 1981 (with the awesome crew of the U.S.S. Peoria LST-1183 Assault Squadron Seven San Diego, CA, and later the TWR-1 Diamond Norfolk, VA.) served with pride and honor. Trained in Japan, Korea, and China, Thailand, Philippines, Australia, Singapore, Hong Kong, Taiwan, (years later returning to Japan to train under Sensei Yamada). In martial arts as well as been a natural heavyweight bodybuilder. Then worked for the U.S. government in Langley, VA. Until 1986. Trained on the weekends at "Vince's gym" in Studio City, CA. under the tutelage of the great trainer Vince Gironda. Later moving to Norfolk, VA. Becoming an accomplished stone mason, building marble and granite mausoleums and installing monuments markers, from Virginia Beach, VA. to North Carolina. Also working with nuclear weapons and reactors, as well as the latest in composite materials for aircraft. During which he traveled around the world over 6 times. Became a diver and retrieved torpedo's for nuclear fast attack submarines on the east coast of the USA. A devoted student to his late $\mathrm{Sifu} \sim \mathrm{Kwan} \mathrm{Li}$, becoming his number one student(and later holding the rank of "Sifu" in both Tai Ch'i Chuan, and Black Dragon Kung Fu(A Southern Style of Hsing-i Chuan or Xing Yi Quan) training for over 45 years in martial arts (specialized in 
Iron palm, and Dragon claw kung). A competitive natural bodybuilder since 1976 who's first contest was in San Deigo, CA 1982 and last contest was in Sacramento, CA. July 22, 2000 "The 2000 INBF Capital City Natural Bodybuilding Championships". Built his first new home in 1985 in Virginia Beach, VA. After getting out of the U.S. Navy active duty. Becoming a freelance writer, and writing technical books and articles for the U.S. Government.

He became an avid treasure hunter/metal detecting both on land and under water, as a hobby, which he still does to this day. Some of his other interests and specialties besides natural bodybuilding, Occult, magic, witchcraft, Taoist sorcery, Development of esoteric powers, Chinese herbalist medicine, certified master of acupressure, rank of Sifu in Tai Ch'i Chuan, and Hsing-i chuan(Xing Yi Quan),World Black Belt Bureau member, Japanese archery (Kyudo, and Kendo), All types of cooking, wine tasting and gardening fresh food, hypnosis, binaural beat-frequency and brain wave activity, animals, artwork for other author's book covers, photography, archeology, rock climbing, drawing, painting, sculpting, woodworking, welding, designing new equipment and devices and exploring distant geographic points of interest and learning about new cultures and customs. The "Black Dragon Ch'i Kung Dojo", is where he will be found teaching students "Ch'i Kung" (Qi Gong), and being a personal trainer to all natural bodybuilders, and his wife Jacqueline in Roy, Utah. USA. ${ }^{1-10}$

\section{Acknowledgements}

None.

\section{Conflict of interest}

The author declares no conflict of interest.

\section{References}

1. Khavinson VK. Peptidergic regulation of aging. Belgium: Humanistica; 2009.

2. Khavinson VK, Neroev VV, Tromimova SV, et al. Unique method for restoration of retinal functions in case of different diseases. Russia: Gerontological Society of the Russian Academy of Sciences; 2011.

3. Khavinson VK. Biologically active food supplements. Russia: Russian Academy of Medical Sciences; 2007.

4. Khavinson VK, Solov YA, Zhilinskii DV, et al. Epigenetic aspects of peptide mediated regulation of aging. Advances in Gerontology. 2012;2(4):277-286.

5. www.pravda.ru/health

6. Khavinson VKh, Bondarev IE, Butyugov AA, et al. Peptide promotes overcoming of the division limit in human somatic cell. Bull Exp Biol Med. 2004;137(5):503-506.

7. http://www.curaderm.net/st-petersburg-institute-of-bioregulationand-gerontology.html

8. Khavinson VK. I think that the small peptides are the best for healthy ageing. Biogerontology. 2012;14(1):1-8.

9. Khavinson VKh, Linkova NS, Polyakova VO, et al. Peptides tissuespecifically stimulates cell differentiation during their aging. Bull Exp Biol Med. 2012;153(1):148-151.

10. Khavinson VKh, Anisimov VN. Peptide regulation of aging: 35-year research experience. Bull Exp Biol Med. 2009;148(1):94-98. 